\title{
A CARTOGRAFIA DO TERRITÓRIO GUARANI NA REGIÃO SUL DO ESTADO DE SANTA CATARINA, BRASIL
}

\author{
THE MAPPING THE TERRITORY GUARANI SOUTH \\ REGION STATE OF SANTA CATARINA, BRASIL
}

Gabriel da Silva Souza

Universidade do Extremo Sul

Catarinense - UNESC, Engenheiro

Agrimensor.

E-mail:

eng.agrimensorgabriel@gmail.com

Juliano Bitencourt Campos

Universidade do Extremo Sul

Catarinense - UNESC, Programa de

Pós-Graduação em Ciências

Ambientais (PPGCA), Laboratório

de Arqueologia Pedro Ignácio

Schmitz (LAPIS).

E-mail: jbi@unesc.net

Nilzo Ivo Ladwig

Universidade do Extremo Sul

Catarinense - UNESC, Programa de

Pós-Graduação em Ciências

Ambientais (PPGCA), Laboratório

de Planejamento e Gestão

Territorial (LabPGT).

E-mail: ladwig@unesc.net

\section{Marcos César Pereira Santos}

Pós-doutorado no Programa de

Pós-Graduação em Geografia e

Núcleo de Estudos

Paleoambientais -

NEPA/UNIOESTE, Campus

Francisco Beltrão, Paraná, Brasil.

Pesquisador associado ao

Laboratório de Aqueologia Pedro

Ignácio Schmitz - LAPIS/UNESC.

E-mail:

marcoscesar.arqueologia@gmail.c om

\section{Resumo}

A arqueologia enquanto disciplina científica não estuda individual e isoladamente seus objetos de análise, mas se utiliza do diálogo com diversas outras ciências para buscar, em conjunto, esclarecer o povoamento do território. No geoprocessamento o SIG é uma das ferramentas, composto por sistemas que integram tecnologia, com o objetivo de armazenar, analisar, manipular e visualizar dados geográficos. Com base nessa linha de raciocínio foi definido como objetivo deste estudo construir uma base de dados referente aos sítios Guarani, com a finalidade de demonstrar a importância do geoprocessamento na documentação para a gestão estratégica do patrimônio arqueológico regional. Foi trabalhado um banco de dados com as informações de 32 sítios arqueológicos Guarani, localizados nos municípios de Balneário Rincão e Araranguá, no sul do Estado de Santa Catarina, de modo que possibilitou a elaboração de mapas temáticos. Os mapas temáticos gerados foram processados com o auxílio do software ArcGis. Os resultados mostraram a importância do SIG como ferramenta de gestão do patrimônio arqueológico, pois permitiu a elaboração de outros mapas temáticos que analisados poderão auxiliar na estratégia de manutenção, conservação dentre outras, além de favorecer o monitoramento e a reavaliação de decisões.

Palavras Chaves: Patrimônio, Arqueologia; Estratégia, Monitoramento; Conservação.
Abstract
Archeology as a scientific discipline does not study its objects of analysis individually and in isolation, but it uses dialogue with several other sciences to seek together clarify the occupation of territory.In geoprocessing the GIS is one of the tools, composed of systems that integrate technology, with the purpose of storing, analyzing, manipulating and visualizing geographic data. Based on this line of reasoning, it was defined as the objective of this study to build a database on the Guarani sites, in order to demonstrate the importance of geoprocessing in the documentation for the strategic management of the regional archaeological heritage. A database was developed with the information of 32 Guarani archaeological sites located in the municipalities of Balneário Rincão and Araranguá, in the south of the State of Santa Catarina, so that it was possible to elaborate thematic maps. The generated thematic maps were processed with the aid of ArcGis software. The results showed the importance of GIS as a tool for the management of archaeological heritage, since it allowed the elaboration of other thematic maps analyzed that could help in the 
Revista Tecnologia e Ambiente, v. 28, 2022, Criciúma, Santa Catarina/SC - ISSN

Eletrônico 2358-9426 e ISSN Impresso 1413-8131

strategy of maintenance, conservation among others, besides favoring the monitoring and reevaluation of decisions.

Keywords: Heritage; Archeology; Strategy; Monitoring; Conservation 
Revista Tecnologia e Ambiente, v. 28, 2022, Criciúma, Santa Catarina/SC - ISSN

Eletrônico 2358-9426 e ISSN Impresso 1413-8131

\section{INTRODUÇÃO}

O significado cultural do sitio arqueológico é determinado pelos valores que a sociedade percebe nele ou em seus elementos. O valor pode ser estético, científico, histórico ou social, ou uma combinação destes. Assim, identificar todos os valores de um lugar, reunir os indivíduos que podem influenciar as decisões que afetam o sitio e obter uma compreensão clara das realidades gerenciais são etapas críticas do processo de planejamento e gestão destes espaços. A informação assim obtida é essencial para o projeto de estratégias de gerenciamento realistas e viáveis. (SULLIVAN, 1995).

Mejuto et al. (2012) afirmam que o geoprocessamento no uso de Sistema Geográfico de Informação (SIG) tem algumas vantagens em comparação com outras tecnologias, por exemplo, os dados são coletados apenas uma vez e mantidos onde podem ser gerenciados de forma mais eficaz. É possível combinar informações espaciais contínuas de diferentes fontes em todo o mundo, compartilhando-a com uma grande quantidade de usuários e aplicativos.

O SIG permite diferentes níveis de detalhe da mesma fonte de dados que pode ser utilizado para pesquisa regional ou geral para fins estratégicos. Outra observação é que o SIG permite desenvolver o compartilhamento de informações espaciais entre as organizações.

Câmara et al. (2001) assinalam que o geoprocessamento se resume às técnicas matemáticas e computacionais, que têm como finalidade tratar as informações geográficas.

As ferramentas que estão inseridas no universo do geoprocessamento são chamadas de sistemas de informação geográfica (SIG). Essas ferramentas nos permitem analisar as informações por meio da interação de várias fontes computacionais e o banco de dados, tornando a representação cartográfica uma das ferramentas mais importantes nesse processo automatizado (CÂMARA et al., 2001).

Aronoff (1989) define SIG como um conjunto, manual ou automatizado, que é utilizado para armazenar e manipular informações georreferenciadas.

Desta forma, a estrutura de um SIG que pretende apoiar a gestão do patrimônio deve conter não somente com uma base de dados com as informações geográficas, mas também variáveis da área de estudo, a fim de proporcionar o cruzamento de informações. O desenvolvimento do SIG no apoio da gestão patrimonial possibilita ao usuário uma 
melhor visão das áreas de interesse, desta forma se tornando uma importante ferramenta na tomada de decisão (LUCCA et al., 2014).

Para melhor gerenciar as informações dos sítios arqueológicos trabalhados por Campos (2015), foi estruturado um banco de dados no modelo relacional em plataforma de SIG, possibilitando o gerenciamento dos dados, a elaboração de mapas temáticos, consultas e manipulação dos sítios registrados, servindo também de referência para registros de sítios posteriores.

A partir da estruturação desse banco de dados georreferenciado em ambiente de SIG, criou-se a possibilidade de análises e outros estudos, com a finalidade de trabalhar a gestão do patrimônio, contribuindo para a conservação e preservação destes espaços, que vêm sendo cada vez mais ameaçados por obras de engenharia.

Desta forma, Souza (2014) afirma que, diante da grande demanda de obras de engenharia, a arqueologia empresarial ou de contrato sofre com a ausência de sistemas de gestão do patrimônio arqueológicos eficientes e com a falta de retorno destas informações à sociedade, evidenciando a necessidade destes mecanismos como ferramenta de gestão.

Mejuto et al. (2012) argumentam que na gestão do patrimônio arqueológico devese estabelecer uma política de gerenciamento com base em documentação e avaliação. $O$ SIG neste processo é importante porque poderá apontar o caminho para a definição de estratégias de manutenção, conservação dentre outras, além de favorecer o monitoramento e a reavaliação de decisões.

Neste contexto, o trabalho tem como objetivo construir uma base de dados referente aos sítios Guarani, com a finalidade de demonstrar a importância do geoprocessamento na documentação para a gestão estratégica do patrimônio arqueológico regional.

\section{2. ÁREA DE ESTUDO, MATERIAIS E MÉTODOS}

\subsection{Localização e descrição da área de estudo}

A área de estudo conforme Figura 1 está inserida no projeto de pesquisa "Arqueologia entre rios: do Urussanga ao Mampituba", desenvolvido pelo grupo de Pesquisa Arqueologia e Gestão Integrada do Território, da Universidade do Extremo Sul Catarinense. 
Figura 1 - Localização da área de estudo
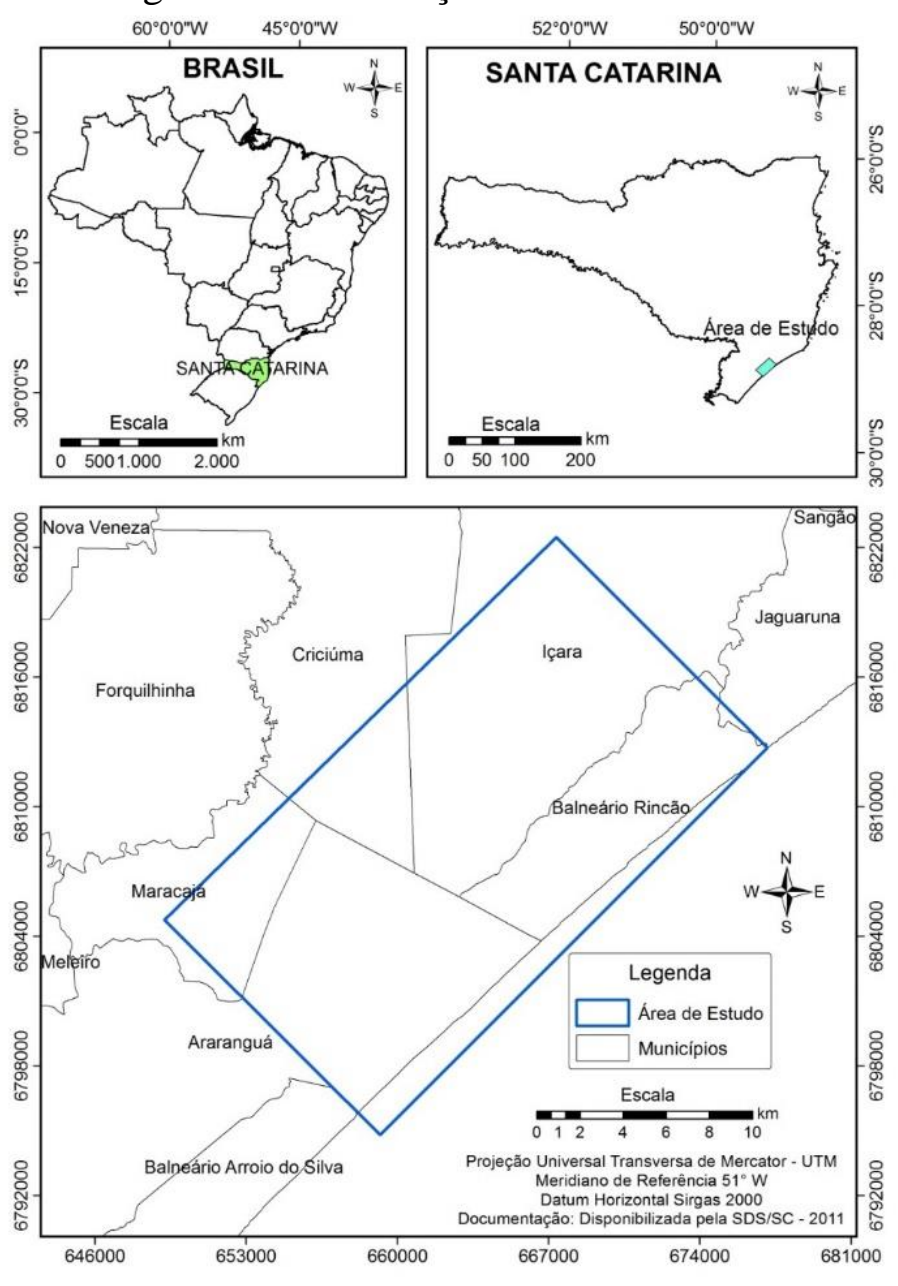

Fonte: autores.

A área possui $353,74 \mathrm{~km}^{2}$ e localiza-se no litoral da região sul do Estado de Santa Catarina, abrangendo os municípios de Araranguá, Balneário Rincão, Criciúma, Içara e Maracajá. Porém, segundo consta na pesquisa de Campos (2015), e seguindo o objetivo do trabalho, verificou-se a existência de sítios arqueológicos da tradição Guarani apenas nos municípios de Araranguá e Balneário Rincão. Desta forma, a área de estudo foi definida entre as seguintes coordenadas UTM (Datum Sirgas 2000, Fuso 22s): ao norte os vértices $6.822 .483(\mathrm{~N})$ e $667.362(\mathrm{E}), 6.812 .728(\mathrm{~N})$ e $677.125(\mathrm{E})$ e; ao sul os vértices $6.804 .765(\mathrm{~N})$ e $649.220(\mathrm{E}), 6.794 .799(\mathrm{~N})$ e $659.196(\mathrm{E})$.

\subsection{Materiais e Métodos}

Para alcançar os objetivos deste trabalho, a metodologia baseou-se em levantamento bibliográfico, análise de documentos, coleta e modelagem dos dados levantados por Campos (2015) e as técnicas de geoprocessamento. 
Campos (2015, p. 112) registrou 54 sítios arqueológicos dentro da área de estudo, 32 deles foram classificados tipologicamente como da tradição Guarani. Desta forma, visando atender aos objetivos estabelecidos, apenas estes 32 sítios foram considerados no estudo, conforme Tabela 1.

Partindo da necessidade de trabalhar a gestão do território, e com a disponibilidade dos dados coletados, foi possível estruturar um banco de dados com a capacidade de gerenciar as informações referentes aos sítios arqueológicos de interesse.

Para atender o primeiro objetivo específico foi necessário gerar uma tabela no software Microsoft Excel com os dados coletados por Campos (2015). Na elaboração da tabela foram definidas as informações relevantes para consulta de cada um dos 32 sítios eleitos para fazer parte do SIG. As informações consideradas na base de dados são: nome, nome popular, coordenadas, município, registro, altitude e área.

Já o segundo objetivo específico, elaboração da estrutura da base de dados em ambiente de SIG, verificou-se a necessidade da utilização do banco de dados no modelo relacional. Além de ser o método mais comum neste tipo de trabalho, o modelo relacional ainda é bastante flexível quanto à manipulação dos dados, organização, redundância e a adição de novas tabelas.

Tabela 1 - Relação dos sítios arqueológicos utilizados no trabalho

\begin{tabular}{|c|c|c|c|c|c|c|c|}
\hline \multirow[b]{2}{*}{ Nome } & \multirow[b]{2}{*}{ Nome Popular } & \multicolumn{2}{|c|}{ Coordenadas } & \multirow[b]{2}{*}{ Município } & \multirow[b]{2}{*}{ Registro } & \multirow[b]{2}{*}{$\begin{array}{c}\text { Altitude } \\
\text { (m) }\end{array}$} & \multirow[b]{2}{*}{$\begin{array}{c}\text { Área } \\
\text { (m) }\end{array}$} \\
\hline & & $\begin{array}{l}\text { Este } \\
(\mathbf{m})\end{array}$ & $\begin{array}{l}\text { Norte } \\
(\mathbf{m})\end{array}$ & & & & \\
\hline $\begin{array}{l}\text { SC-ARA- } \\
018\end{array}$ & Aldeia da Balsa & 658.364 & 6.799 .056 & Araranguá & 1998 & 10 & $40 \times 50$ \\
\hline $\begin{array}{l}\text { SC-ARA- } \\
003\end{array}$ & $\begin{array}{l}\text { Aldeia do } \\
\text { Marcelino }\end{array}$ & 663.838 & 6.805 .800 & $\begin{array}{c}\text { Bal. } \\
\text { Rincão }\end{array}$ & 1998 & 10 & $20 \times 25$ \\
\hline $\begin{array}{l}\text { SC-ARA- } \\
004\end{array}$ & $\begin{array}{l}\text { Aldeia da } \\
\text { Lagoa Mãe } \\
\text { Luzia }\end{array}$ & 663.557 & 6.805 .711 & $\begin{array}{l}\text { Bal. } \\
\text { Rincão }\end{array}$ & 1999 & 15 & $45 \times 50$ \\
\hline $\begin{array}{l}\text { SC-ARA- } \\
005\end{array}$ & $\begin{array}{l}\text { Aldeia do } \\
\text { Levandoski }\end{array}$ & 657.746 & 6.800 .919 & Araranguá & 1998 & 12 & $30 \times 40$ \\
\hline $\begin{array}{l}\text { SC-ARA- } \\
006\end{array}$ & $\begin{array}{c}\text { Aldeia da Roça } \\
\text { de Melancia }\end{array}$ & 662.715 & 6.803 .941 & Araranguá & 1998 & 15 & $50 \times 50$ \\
\hline $\begin{array}{l}\text { SC-ARA- } \\
007\end{array}$ & $\begin{array}{c}\text { Aldeia da Roça } \\
\text { de Milho }\end{array}$ & 659.992 & 6.801 .688 & Araranguá & 1998 & 15 & $50 \times 40$ \\
\hline $\begin{array}{l}\text { SC-ARA- } \\
008\end{array}$ & $\begin{array}{c}\text { Campo Mãe } \\
\text { Luzia } 1\end{array}$ & 660.532 & 6.802 .444 & Araranguá & 2005 & 15 & $30 \times 40$ \\
\hline $\begin{array}{l}\text { SC-ARA- } \\
009\end{array}$ & $\begin{array}{c}\text { Campo Mãe } \\
\text { Luzia } 2\end{array}$ & 660.665 & 6.802 .586 & Araranguá & 2005 & 15 & $20 \times 30$ \\
\hline
\end{tabular}




\begin{tabular}{|c|c|c|c|c|c|c|c|}
\hline \multirow[b]{2}{*}{ Nome } & \multirow[b]{2}{*}{ Nome Popular } & \multicolumn{2}{|c|}{ Coordenadas } & \multirow[b]{2}{*}{ Município } & \multirow[b]{2}{*}{ Registro } & \multirow{2}{*}{$\begin{array}{l}\text { Altitude } \\
\quad \text { (m) }\end{array}$} & \multirow{2}{*}{$\begin{array}{l}\text { Área } \\
\text { (m) }\end{array}$} \\
\hline & & $\begin{array}{l}\text { Este } \\
(\mathrm{m})\end{array}$ & $\begin{array}{l}\text { Norte } \\
\text { (m) }\end{array}$ & & & & \\
\hline $\begin{array}{l}\text { SC-ARA- } \\
010\end{array}$ & $\begin{array}{l}\text { Campo Mãe } \\
\text { Luzia } 3\end{array}$ & 661.021 & 6.802 .958 & Araranguá & 2005 & 20 & $\begin{array}{c}100 \mathrm{x} \\
100\end{array}$ \\
\hline $\begin{array}{l}\text { SC-ARA- } \\
011\end{array}$ & $\begin{array}{l}\text { Campo Mãe } \\
\text { Luzia } 4\end{array}$ & 661.354 & 6.803 .135 & Araranguá & $\begin{array}{l}\text { Sem } \\
\text { Ident. }\end{array}$ & 15 & $65 \times 55$ \\
\hline $\begin{array}{l}\text { SC-ARA- } \\
016\end{array}$ & $\begin{array}{c}\text { Aldeia } \\
\text { Luquinha do Zé } \\
\text { Pequeno }\end{array}$ & 668.478 & 6.809 .435 & $\begin{array}{l}\text { Bal. } \\
\text { Rincão }\end{array}$ & 1998 & 18 & $20 \times 20$ \\
\hline $\begin{array}{l}\text { SC-ARA- } \\
017\end{array}$ & SC-IÇ-02 & 665.572 & 6.805 .042 & $\begin{array}{l}\text { Bal. } \\
\text { Rincão }\end{array}$ & 1998 & 30 & $10 \times 20$ \\
\hline $\begin{array}{l}\text { SC-ARA- } \\
002\end{array}$ & $\begin{array}{c}\text { Aldeia da } \\
\text { Escola Lagoa } \\
\text { dos Esteves }\end{array}$ & 666.277 & 6.808 .413 & $\begin{array}{l}\text { Bal. } \\
\text { Rincão }\end{array}$ & 1998 & 40 & $30 \times 25$ \\
\hline $\begin{array}{l}\text { SC-ARA- } \\
019\end{array}$ & $\begin{array}{l}\text { Aldeia do } \\
\text { Arseno }\end{array}$ & 667.245 & 6.808 .984 & $\begin{array}{l}\text { Bal. } \\
\text { Rincão }\end{array}$ & 1998 & 28 & $40 \times 25$ \\
\hline $\begin{array}{l}\text { SC-ARA- } \\
020\end{array}$ & $\begin{array}{l}\text { Aldeia do } \\
\text { Campestre } \\
\text { Aldeia do }\end{array}$ & 666.999 & 6.808 .984 & $\begin{array}{l}\text { Bal. } \\
\text { Rincão }\end{array}$ & 1998 & 5 & $30 \times 35$ \\
\hline $\begin{array}{l}\text { SC-ARA- } \\
021\end{array}$ & $\begin{array}{l}\text { Cemitério da } \\
\text { Lagoa dos } \\
\text { Esteves }\end{array}$ & 665.644 & 6.807 .591 & $\begin{array}{l}\text { Bal. } \\
\text { Rincão }\end{array}$ & 1998 & 70 & $50 \times 45$ \\
\hline $\begin{array}{l}\text { SC-ARA- } \\
022\end{array}$ & $\begin{array}{l}\text { Aldeia do } \\
\text { Mussuline }\end{array}$ & 665.312 & 6.807 .103 & $\begin{array}{l}\text { Bal. } \\
\text { Rincão }\end{array}$ & 1998 & 40 & $40 \times 50$ \\
\hline $\begin{array}{l}\text { SC-ARA- } \\
023\end{array}$ & $\begin{array}{l}\text { Aldeia do } \\
\text { Pomar }\end{array}$ & 665.481 & 6.807 .266 & $\begin{array}{l}\text { Bal. } \\
\text { Rincão }\end{array}$ & 1998 & 60 & $20 \times 35$ \\
\hline $\begin{array}{l}\text { SC-ARA- } \\
024\end{array}$ & $\begin{array}{c}\text { Aldeia do } \\
\text { Camping Silva }\end{array}$ & 665.425 & 6.806 .670 & $\begin{array}{l}\text { Bal. } \\
\text { Rincão }\end{array}$ & 1998 & 10 & $30 \times 35$ \\
\hline $\begin{array}{l}\text { SC-ARA- } \\
027\end{array}$ & $\begin{array}{c}\text { Aldeia do } \\
\text { Camping Viana }\end{array}$ & 665.314 & 6.806 .193 & $\begin{array}{l}\text { Bal. } \\
\text { Rincão }\end{array}$ & 1998 & 10 & $30 \times 45$ \\
\hline $\begin{array}{l}\text { SC-ARA- } \\
028\end{array}$ & $\begin{array}{l}\text { Acampamento } \\
\text { da Plataforma } \\
\text { da Barra Velha }\end{array}$ & 668.977 & 6.807 .260 & $\begin{array}{l}\text { Bal. } \\
\text { Rincão }\end{array}$ & 1998 & 10 & $30 \times 40$ \\
\hline $\begin{array}{l}\text { SC-URU- } \\
002\end{array}$ & Pedreira & 671.920 & 6.812 .157 & $\begin{array}{l}\text { Bal. } \\
\text { Rincão }\end{array}$ & 2003 & 12 & $15 \times 15$ \\
\hline $\begin{array}{l}\text { SC-URU- } \\
001\end{array}$ & $\begin{array}{l}\text { Urussanga } \\
\text { Velha }\end{array}$ & 673.150 & 6.814 .732 & $\begin{array}{l}\text { Bal. } \\
\text { Rincão }\end{array}$ & 2003 & 20 & $20 \times 45$ \\
\hline $\begin{array}{l}\text { SC-ARA- } \\
031\end{array}$ & Sem Ident. & 660.900 & 6.798 .650 & $\begin{array}{l}\text { Bal. } \\
\text { Rincão }\end{array}$ & 2011 & 3 & $\begin{array}{c}100 x \\
80\end{array}$ \\
\hline $\begin{array}{l}\text { SC-ARA- } \\
034\end{array}$ & Mauro Inácio & 657.453 & 6.808 .024 & Araranguá & $\begin{array}{l}\text { Sem } \\
\text { Ident. }\end{array}$ & 46 & $\begin{array}{c}1000 \\
\mathrm{~m}^{2}\end{array}$ \\
\hline $\begin{array}{l}\text { SC-ARA- } \\
036\end{array}$ & Sem Ident. & 673.612 & 6.812 .180 & $\begin{array}{l}\text { Bal. } \\
\text { Rincão }\end{array}$ & $\begin{array}{l}\text { Sem } \\
\text { Ident. }\end{array}$ & 17 & $30 \times 35$ \\
\hline $\begin{array}{l}\text { SC-ARA- } \\
037\end{array}$ & Sem Ident. & 668.218 & 6.808 .357 & $\begin{array}{l}\text { Bal. } \\
\text { Rincão }\end{array}$ & $\begin{array}{l}\text { Sem } \\
\text { Ident. }\end{array}$ & 5 & $25 \times 30$ \\
\hline $\begin{array}{l}\text { SC-ARA- } \\
039\end{array}$ & Sem Ident. & 668.818 & 6.810 .605 & $\begin{array}{l}\text { Bal. } \\
\text { Rincão }\end{array}$ & $\begin{array}{l}\text { Sem } \\
\text { Ident. }\end{array}$ & 80 & $30 \times 50$ \\
\hline
\end{tabular}




\begin{tabular}{|c|c|c|c|c|c|c|c|}
\hline \multirow[b]{2}{*}{ Nome } & \multirow[b]{2}{*}{ Nome Popular } & \multicolumn{2}{|c|}{ Coordenadas } & \multirow[b]{2}{*}{ Município } & \multirow[b]{2}{*}{ Registro } & \multirow[b]{2}{*}{$\begin{array}{l}\text { Altitude } \\
\text { (m) }\end{array}$} & \multirow[b]{2}{*}{$\begin{array}{l}\text { Área } \\
\text { (m) }\end{array}$} \\
\hline & & $\begin{array}{l}\text { Este } \\
(\mathbf{m})\end{array}$ & $\begin{array}{l}\text { Norte } \\
\text { (m) }\end{array}$ & & & & \\
\hline $\begin{array}{l}\text { SC-ARA- } \\
001\end{array}$ & $\begin{array}{l}\text { Zulemar Maria } \\
\text { de Souza }\end{array}$ & 666.211 & 6.808 .846 & $\begin{array}{c}\text { Bal. } \\
\text { Rincão }\end{array}$ & 2013 & 60 & $30 \times 40$ \\
\hline $\begin{array}{l}\text { SC-ARA- } \\
049\end{array}$ & Sem Ident. & 659.413 & 6.797 .608 & Araranguá & $\begin{array}{l}\text { Sem } \\
\text { Ident. }\end{array}$ & 12 & $30 \times 30$ \\
\hline $\begin{array}{l}\text { SC-ARA- } \\
051\end{array}$ & Sem Ident. & 658.281 & 6.797 .554 & Araranguá & $\begin{array}{l}\text { Sem } \\
\text { Ident. }\end{array}$ & 20 & $10 \times 15$ \\
\hline $\begin{array}{l}\text { SC-ARA- } \\
052\end{array}$ & $\begin{array}{c}\text { Lagoa dos } \\
\text { Bichos }\end{array}$ & 657.931 & 6.796 .997 & Araranguá & $\begin{array}{l}\text { Sem } \\
\text { Ident. }\end{array}$ & 15 & $80 \times 80$ \\
\hline
\end{tabular}

Fonte: Adaptado de Campos (2015).

A etapa de relação dos dados junto ao software de geoprocessamento utlizado só foi possivel com a adequação dos dados organizados no software Microsoft Excel para o software Microsoft Access, devido ao seu melhor desempenho e relação com o software ArcMap. Assim toda a modelagem e o armazenamento do banco de dados foi desenvolvido no software ArcMap 10.2.1 de produção da Esri.

Por fim, no terceiro objetivo específico, com os dados já adequados e ligados ao ambiente de SIG, buscou-se a elaboração de produtos cartográficos com potencial para o auxílio na gestão do patrimônio arqueológico, sendo a cartografia temática uma importante forma de apresentação dos resultados.

O sistema de projeção adotado no ambiente de SIG para a elaboração de todo o trabalho foi o sistema UTM (Universal Transversa de Mercator) e o Datum utilizado SIRGAS 2000 (Sistema de Referência Geocêntrico para as Américas).

Diante do que foi proposto acima, segue abaixo (Quadro 1) um resumo da relação dos objetivos específicos com os procedimentos metodológicos desenvolvidos neste trabalho.

Quadro 1 - Relação entre os objetivos específicos e os procedimentos metodológicos

\begin{tabular}{|l|lr|}
\hline OBJETIVOS ESPECÍFICOS & \multicolumn{2}{c|}{ PROCEDIMENTOS METODOLÓGICOS } \\
\hline $\begin{array}{l}\text { Definir as variáveis e } \\
\text { elaborar uma base de dados } \\
\text { espacial e alfanuméricos em } \\
\text { ambiente de SIG. }\end{array}$ & $\begin{array}{l}\checkmark \\
\text { Realizar pesquisa bibliográfica, análise de } \\
\text { Campos (2015) mencionado acima; } \\
\checkmark \quad \text { Gerar e organizar uma tabela no software } \\
\text { Microsoft Excel com os dados levantados; }\end{array}$ \\
\hline
\end{tabular}




\begin{tabular}{|c|c|}
\hline $\begin{array}{l}\text { - Estruturar um } \quad \text { SIG } \\
\text { aplicado à gestão do patrimônio } \\
\text { arqueológico. }\end{array}$ & $\begin{array}{l}\checkmark \quad \text { Adequar a tabela de dados para o formato } \\
\text { do software Microsoft Access; } \\
\checkmark \quad \text { Relacionar a base de dados ao software } \\
\text { ArcMap por meio do modelo relacional; } \\
\checkmark \quad \text { Utilizar as técnicas de geoprocessamento } \\
\text { no ambiente do software ArcMap; }\end{array}$ \\
\hline $\begin{array}{l}\text { - Elaborar mapas temáticos, } \\
\text { a fim de auxiliar na análise dos } \\
\text { elementos que fazem parte do } \\
\text { patrimônio arqueológico dos } \\
\text { sítios. }\end{array}$ & $\begin{array}{l}\checkmark \quad \text { Elaborar produtos e modelos que possam } \\
\text { ser aplicados à gestão do patrimônio arqueológico } \\
\text { e à tomada de decisões, a partir do SIG. }\end{array}$ \\
\hline
\end{tabular}

Fonte: Elaborado pelo autor.

\section{RESULTADOS}

A partir da pesquisa bibliográfica e coleta de dados já discutida e desenvolvida no trabalho, chegou-se aos resultados aqui apresentados.

No ambiente do software Microsoft Excel, foi trabalhada a tabulação dos sítios registrados por Campos (2015), configurando a mesma no modelo de banco de dados relacional.

Por meio do modelo relacional foi importado no software ArcMap o banco de dados, fornecendo as informações e atributos pré-estabelecidos referentes a cada um dos sítios arqueológicos Guarani.

O modelo do banco de dados relacional se mostrou eficiente no trabalho desenvolvido, possibilitando qualquer tipo de modificação e atualização do banco de dados. É importante salientar que novos atributos podem ser inseridos nos sítios arqueológicos já existentes, bem como a locação de novos e a ampliação da área de estudo.

A utilização do banco de dados em conjunto com outras bases cartográficas pode gerar inúmeros mapas temáticos, com a finalidade de analisar e apresentar os resultados do SIG, neste caso aplicado à gestão dos sítios arqueológicos. Partindo desta necessidade de gestão, foram gerados três produtos cartográficos: mapa de localização dos sítios Guarani e mapa de ameaça antrópica.

O mapa de localização dos sítios Guarani foi elaborado utilizando os dados de Campos (2015) e as Ortofotos da Secretaria de Estado do Desenvolvimento sustentável - 
SDS (2010). Esse mapa temático tem como objetivo identificar as informações referentes aos sítios, sendo essas, a localização geográfica, o nome dos sítios, data de registro, altitude média em relação ao nível do mar, sua tradição (neste caso a tradição Guarani) e a sua área de distribuição espacial. Os traçados das vias próximas aos sítios representados no mapa foram vetorizados a partir da interpretação das ortofotos de 2010 , neste caso serviram também para o estudo de impacto que as estradas e rodovias têm em relação aos sítios. Isto porque as estradas e rodovias são as principais vias de expansão urbana. Verificou-se também que dos 32 sítios arqueológicos, 20 deles se encontram no município de Balneário Rincão e os outros 12 no município de Araranguá.

O mapa da Figura 2 representa a histórica relação entre a ocupação Guarani e a hidrografia na área de estudo.

A este respeito Noelli (1999/2000, p. 255) argumenta que: A maioria dos sítios arqueológicos está próxima de cursos d'água piscosos e, para pescar, conforme as fontes históricas, os Guarani dominavam pelo menos 11 técnicas distintas. 1) Nas corredeiras usavam o pari, armadilha com uma barreira que conduz os peixes para um cesto que os captura em quantidades controladas; 2) Nas águas mansas espalhavam ictiotóxicos. Os grupos que viviam à beira-mar ou em cursos d'água que nele desaguavam utilizavam intensamente seus recursos, como as piracemas e a coleta intensa de moluscos.

O mapa então reforça a evidencia de que as populações Guarani eram bastante ligadas aos recursos hídricos. Isto é confirmado pela maior densidade de sítios arqueológicos no entorno dos rios e lagoas, bem como na proximidade dos mesmos.

A zona costeira atualmente representa geograficamente a área mais densamente habitada no Brasil, por essa questão surgiu a necessidade de analisar os riscos que esses sítios estão expostos.

A Figura 3 apresenta o mapa de ameaças antrópicas. Resultado do cruzamento dos dados e do mapeamento de uso e cobertura do solo.

Este mapa teve como finalidade identificar a proximidade dos sítios arqueológicos com as ações antrópicas de maior ameaça. Foram definidas as fontes ativas de extração mineral de areia ou argila e a urbanização como os principais fatores de risco aos sítios arqueológicos, desta forma avaliando a integridade e qual tipo de ação danificou o mesmo. 
Assim, pode-se dizer que o banco de dados, em conjunto com as diversas bases cartográficas utilizadas foram fundamentais para se chegar aos resultados discutidos, sendo que o modelo relacional se adaptou perfeitamente ao software utilizado e aos produtos cartográficos gerados. Outra peculiaridade do banco de dados dentro de um SIG é armazenar, manipular, modelar e cruzar os dados.

Figura 2 - Mapa de localização dos sítios arqueológicos Guarani

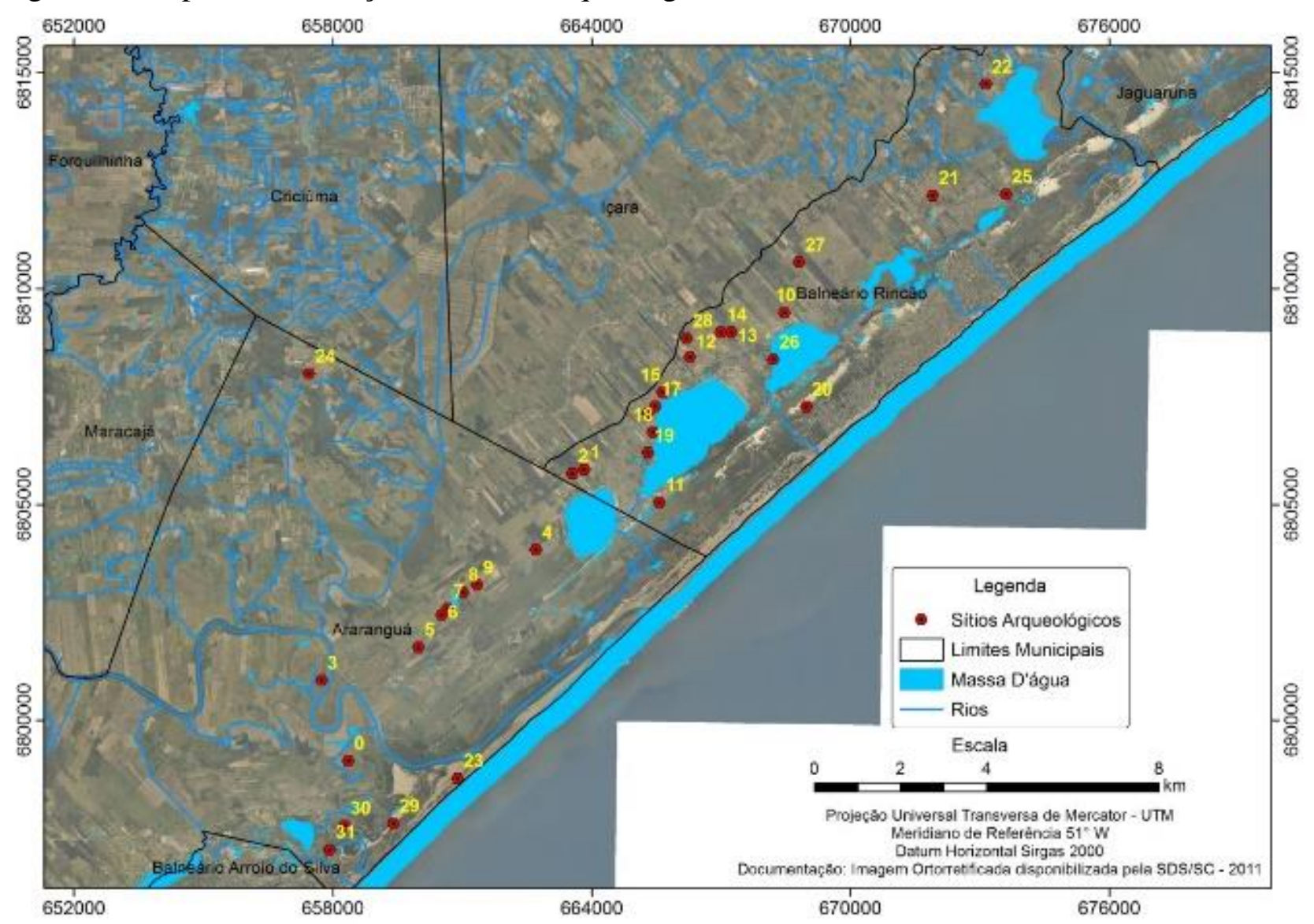

O mapa da figura pode ser encontrado em apêndice em uma escala maior.

Fonte: autores

O armazenamento permitirá adicionar novos sítios arqueológicos e polígonos de estudo se necessário, para que se possa manter o cadastro sempre atualizado, servindo também de referência para órgãos de interesse em patrimônio arqueológico. Já a manipulação nos possibilita alterar dados sem comprometer o seu histórico, adicionando novos atributos a dados já existentes.

O banco de dados relacional se destaca em relação aos outros métodos por utilizar várias tabelas que se organizam em matrizes, isto nos permite, em ambiente de SIG, adicionar novas linhas e colunas. 
Partindo da premissa de se trabalhar apenas os sítios arqueológicos Guarani como objeto de pesquisa, vale reforçar que neste trabalho foram adotados apenas 32 sítios de todos os 54 registrados por Campos (2015), o que demonstra a possibilidade de se trabalhar com uma quantidade ainda maior de dados, tornando o SIG ainda mais interessante.

Figura 3 - Mapa de ameaça antrópica aos sítios arqueológicos Guarani

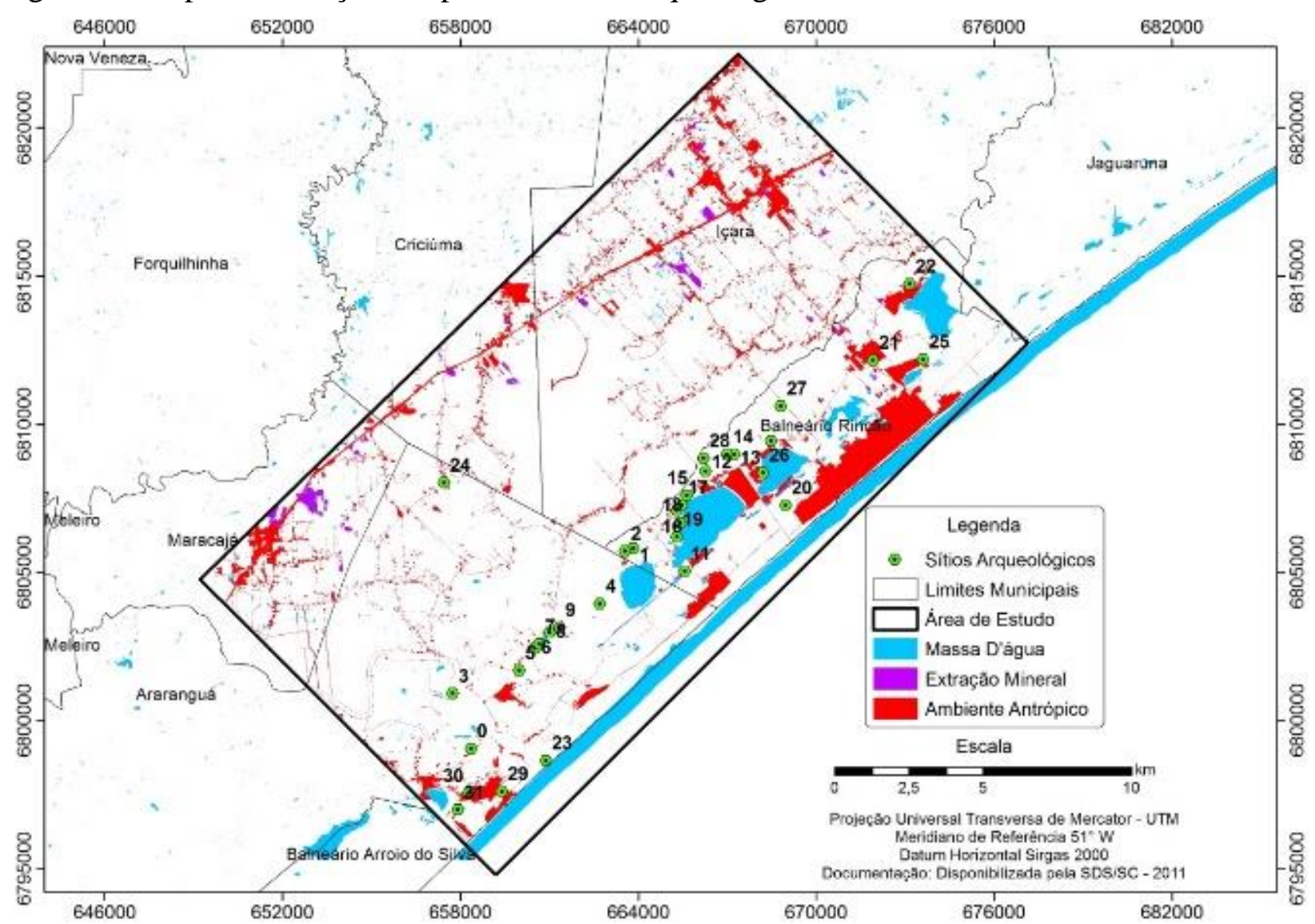

O mapa da figura pode ser encontrado em apêndice em uma escala maior.

Fonte: autores

Quanto aos mapas temáticos elaborados, de forma geral se mostraram bastante eficientes para a representação dos objetivos propostos. Estes resultaram em um mapa de localização, com as informações e a localização geográfica do objeto de interesse no estudo, no mapa hidrográfico da área de estudo, que se constituiu em uma importante ferramenta de pesquisa, reforçando a grande concentração de sítios Guarani próximos aos recursos hídricos; e no mapa de ameaças antrópicas representando cartograficamente as áreas urbanizadas e de extração mineral de todo o polígono, espacializando a ameaça que cada um dos sítios está exposto. 
Revista Tecnologia e Ambiente, v. 28, 2022, Criciúma, Santa Catarina/SC - ISSN

Eletrônico 2358-9426 e ISSN Impresso 1413-8131

\section{CONSIDERAÇÕES FINAIS}

Sabe-se que é da natureza humana ocupar espaços de fácil acesso aos bens naturais, pois estes locais nos oferecem as melhores condições de vida e sobrevivência. Desta forma entende-se que todo o litoral (hoje a região mais densamente povoada do país), foi inicialmente ocupado por grupos pré-coloniais e posteriormente por grupos coloniais. Partindo desta linha de raciocínio, a região litorânea se destaca por apresentar um alto potencial de patrimônios arqueológicos.

Trabalhar o SIG em conjunto com o potencial arqueológico e outras fontes de informações torna-se uma ferramenta fundamental na conservação e preservação destes patrimônios, que nos contam um pouco do nosso passado.

A utilização do SIG como ferramenta na gestão do patrimônio arqueológico se mostrou eficiente, uma vez que ao trabalhar com os dados georreferenciados em conjunto com outras fontes cartográficas podemos chegar a vários resultados, e por fim enriquecer as discussões do objeto de estudo.

Todo o trabalho foi desenvolvido dentro da metodologia de geoprocessamento que engloba uma gama de geotecnologias, que de forma direta ou indireta contribuíram para os resultados deste trabalho. A dinâmica com que se trabalhou o SIG mostrou as suas ferramentas de análise e cruzamento de dados, além da satisfatória relação com o banco de dados, buscando a melhor forma de representar os dados em estudo.

O banco de dados, de maneira geral, se mostrou uma ferramenta importante para a gestão dos sítios arqueológicos pela sua fácil aplicação em ambiente de SIG e interatividade com o usuário, apresentando para a comunidade em geral a necessidade de conscientização da conservação e preservação dos sítios arqueológicos.

A aplicação do SIG em um determinado polígono representou muito pouco, visto que poderíamos trabalhar com um polígono maior, ou até mesmo a nível federal, ajudando a responder várias questões quanto ao patrimônio arqueológico que está a nossa volta. $\mathrm{O}$ trabalho mostrou que quanto maior a base dos dados em ambiente de SIG mais consistentes podem ser os resultados. Pode-se, ainda, estudar outras áreas de pesquisa em busca de mais informações.

O estudo desenvolvido em ambiente de SIG proporcionou uma melhor percepção do espaço que nos cerca, e mostrou que para gerir o mesmo é necessário trabalhar com as mais diversas ferramentas do geoprocessamento, sendo o SIG a mais importante nesse 
processo em que é necessário traçar ações estratégicas de conservação do patrimônio arqueológico ameaçado.

Preparar um plano de gestão é proteger e conservar o significado cultural dos sítios através de decisões apropriadas de gerenciamento. O plano deverá destinar-se a implementar uma série de ações de proteção que possa impedir ou retardar a deterioração do sítio, seja essa deterioração física ou, em vez disso, se relacionar com a perda de outros valores culturais.

Por meio do trabalho de pesquisa desenvolvido em ambiente de SIG fica evidenciado que a atuação do Engenheiro Agrimensor em equipes multidisciplinares vem sendo cada vez mais frequente, desta forma contribuindo no planejamento e na gestão territorial de espaços que abrigam patrimônio arqueológico.

\section{REFERÊNCIAS}

ARONOFF, S. Geographic Information Systems: a management perspective. WDL Publications, Ottawa, Canadá, 1989.

CÂMARA, G.; DAVIS, C.; MONTEIRO, A. M. V. Introdução à Ciência da Geoinformação. [on-line] 2001. Disponível em: <http://www.dpi.inpe.br/gilberto/livro/introd/>. Acesso em: 30 de ago. 2014.

CAMPOS, J. B. Arqueologia Entre Rios e a Gestão Integrada do Território no Extremo Sul de Santa Catarina - Brasil. 2015. Tese (Doutorado) - Universidade de Trás-os-Montes e Alto Douro, Portugal, 2015.

LADWIG, N. I.; SCHWALM, H. (Org.). Gestão socioambiental das cidades no Século XXI: Teorias, Conflitos e Desafios. Florianópolis: Editora Insular, 2013, p. 209-214.

LUCCA, A. F.; SANTOS, M. C. P.; CAMPOS, J. B.; ZOCCHE, J. J. Sistema de Informações Geográficas Aplicado a Gestão do Patrimônio Arqueológico na Região Litorânea dos Municípios de Araranguá e Içara - SC. In: CAMPOS, J. B.; ZOCCHE, J. J.; CEREZER, J, F.; OOSTERBEEK, L. M. Arqueologia Ibero-Americana e Transatlântica: Arqueologia, Sociedade e Território. Erechim, RS: Habilis, 2014.

MEJUTO, J.; GÓMEZ CASTAÑO, J.; RODRÍGUEZ-CADEROT G. GIS Techniques in Archaeology: An Archaeoastronomical Approach. Archaeology, New Approaches in Theory and Techniques. Dr. Imma Ollich-Castanyer (Ed.), 2012, ISBN: 978-953-510590-9, InTech, Available from: <http://www.intechopen.com/books/archaeology-newapproaches-in-theory-and-techniques/gis-techniques-inarchaeology-anarchaeoastronomical-approach>.

NOELLI, F. S. A ocupação humana na região sul do Brasil: Arqueologia, debates e perspectivas - 1872/2000. Revista USP, São Paulo, n. 44, 1999-2000, p. 218-269.

SOUZA, A. S. Geoprocessamento Aplicado no Estudo de Sítios Arqueológicos: O Caso Mãe Luzia I e Mãe Luzia II Município de Araranguá - SC. 2014. TCC (Graduação) 
Revista Tecnologia e Ambiente, v. 28, 2022, Criciúma, Santa Catarina/SC - ISSN

Eletrônico 2358-9426 e ISSN Impresso 1413-8131

- Universidade do Extremo Sul Catarinense, Criciúma, 2014.

SULLIVAN, S. A Planning Model for the Management of Archaeological Sites. In: TORRE, M. De La. The Conservation of Archaeological Sites in the Mediterranean Region. An International Conference Organized by the Getty Conservation Institute and the Paul Getty Museum. 1995, p.15-26. Disponível em: <http://d2aohiyo3d3idm.cloudfront.net/publications/virtuallibrary/0892364866.pdf>. Acesso em: 30 de maio. 2017. 
Revista Tecnologia e Ambiente, v. 28, 2022, Criciúma, Santa Catarina/SC - ISSN Eletrônico 2358-9426 e ISSN Impresso 1413-8131

Figura 2 - Mapa de localização dos sítios arqueológicos Guarani

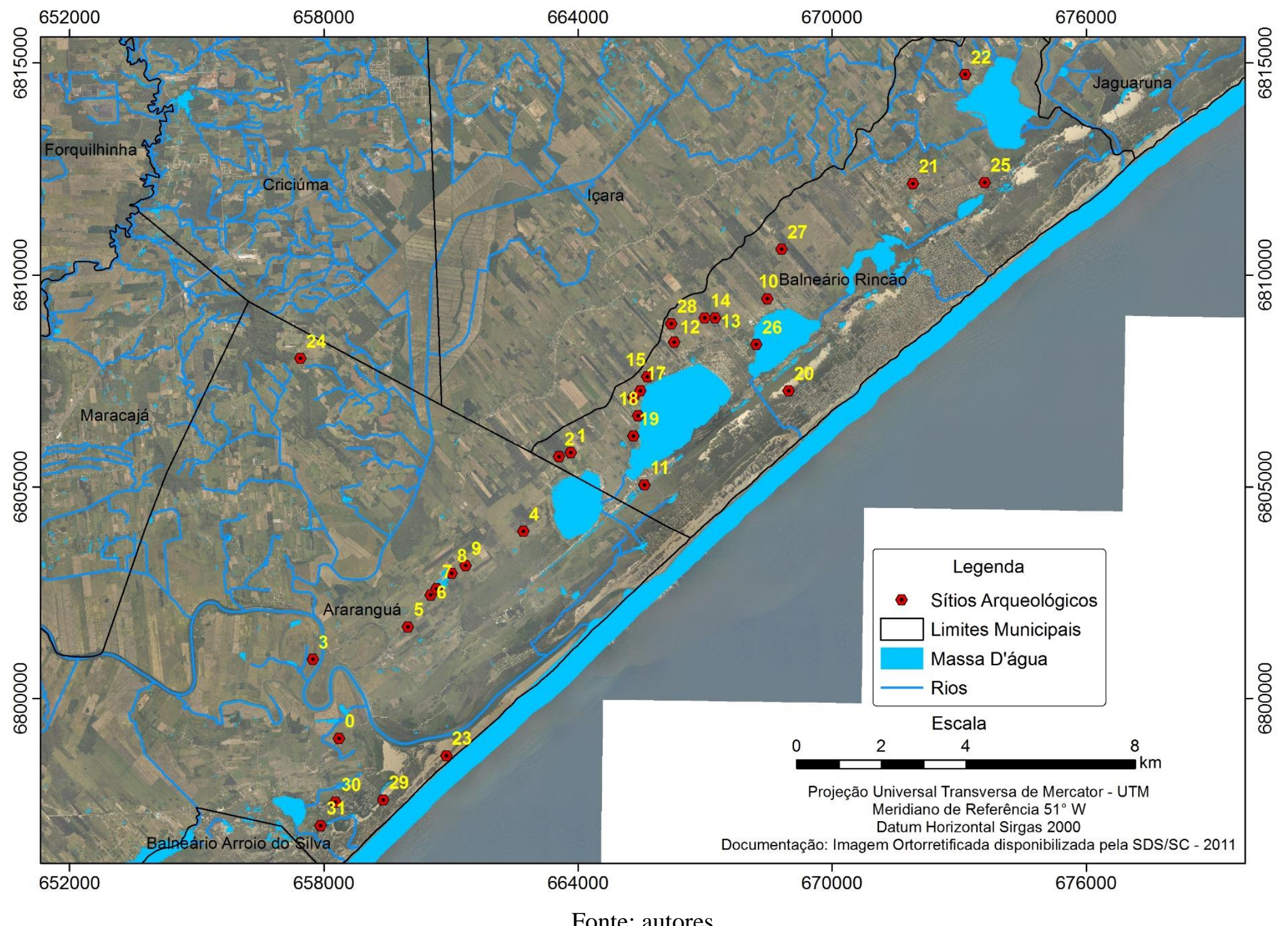


Revista Tecnologia e Ambiente, v. 28, 2022, Criciúma, Santa Catarina/SC - ISSN Eletrônico 2358-9426 e ISSN Impresso 1413-8131

Figura 3 - Mapa de ameaça antrópica aos sítios arqueológicos Guarani

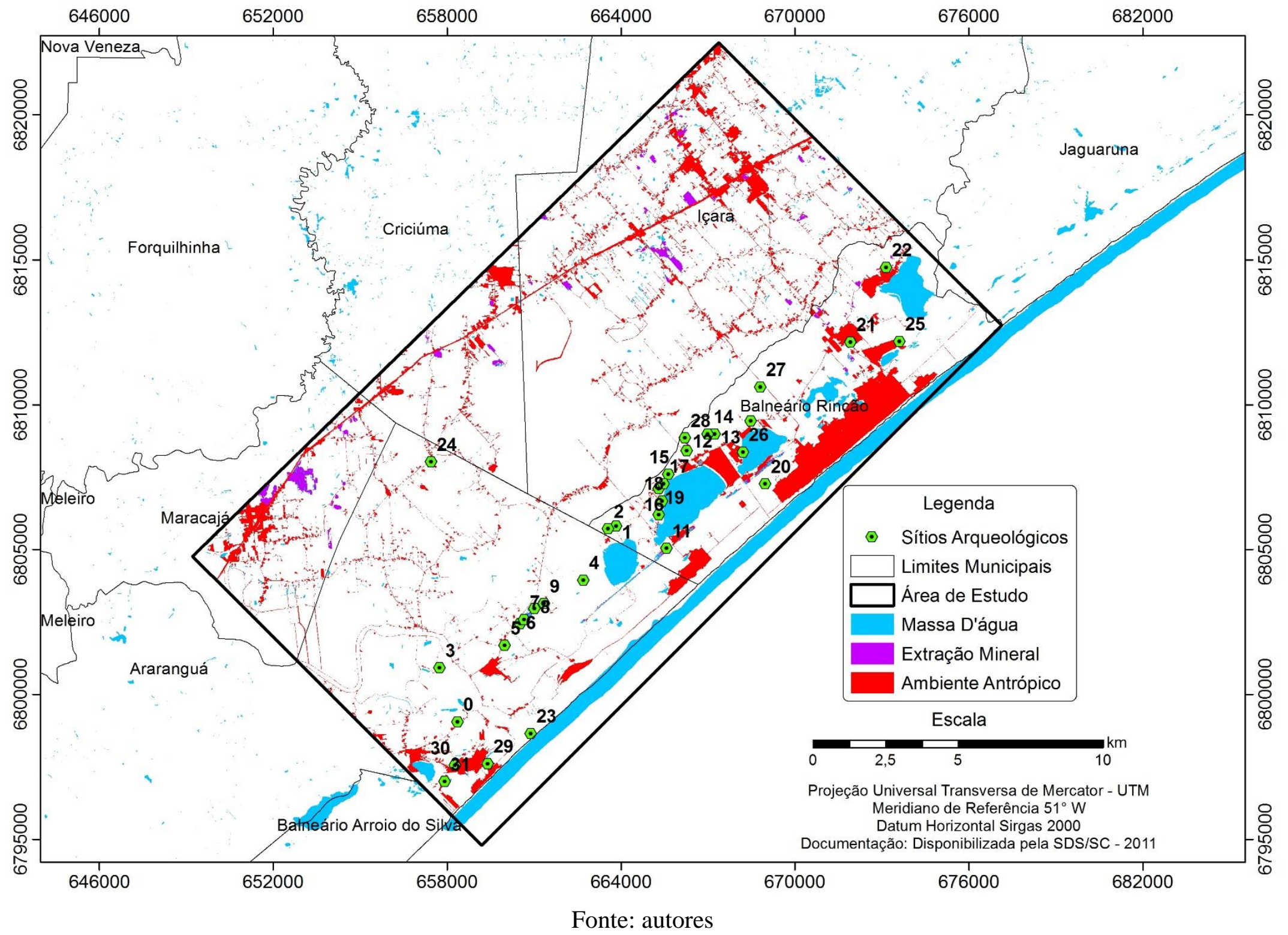

